\title{
Space Charge Dynamics in Polyimide Based Nanocomposites
}

\author{
Junwei ZHA, Zhimin DANG \\ College of Materials Science and Engineering \\ Beijing University of Chemical Technology \\ Beijing, China \\ dangzm@mail.buct.edu.cn
}

\author{
George CHEN \\ School of Electronics and computer \\ University of Southampton \\ Southampton, United Kingdom \\ gc@ecs.soton.ac.uk
}

\begin{abstract}
Polymer nanocomposites have attracted wide interest as a method of enhancing polymer properties and extending their applications. In polymer nanocomposites, chemically dissimilar components are combined at the nanometer scale, and stronger interactions between the polymer and nanoparticles produce markedly improved materials with better electrical, mechanical, thermal, and rheological performances than the conventional filled polymer composites. In recent years, polymer nanocomposites have been extensively studied in optical, thermal and mechanical properties, but there has been relatively little research into dielectric properties. Polyimide is an excellent dielectric material that has a wide range of applications at elevated temperatures. In this paper, space charge in polyimide based nanocomposites has been measured under high dc electric fields using the pulsed electroacoustic technique. Different types and levels of nanoparticles have been studied to understand the interaction between nanoparticles and polyimide matrix. Charge dynamics during "volts on" and "volts off" have shown that the addition of nanoparticles has a significant influence on charge formation and decay processes.
\end{abstract}

Keywords-space charge; electrical properties; polyimide; nanocomposite

\section{INTRODUCTION}

Polyimide (PI), one of the versatile engineering materials, has wide applications because of its good dielectric properties, high thermal and chemical stability, and high electrical breakdown strength. Therefore, it is often used as a promising matrix material for nanocomposites [1-2]. With the development of new electrical technology, the traditional materials have been challenged under some special working conditions and thus more new functional materials are being propounded. One of the new potential applications is to incorporate nanoparticles into a polymer matrix to improve the dielectric properties, which are very important in electrical and electronics industries [3-4]. However, most of research work was studied about thermal and dielectric properties of the nanocpomosites while little attention has been paid to space charge dynamics in polyimide nanocomposites.

Space charge distribution in dielectric materials under dc electric stress has been studied over the last few decades thanks to the invention of several non-destructive techniques. It has been found that space charges can have a profound influence on the degradation or ageing [5-6]. These space charges were introduced by either the injection of charge carriers from the electrodes into the materials or ionization of the nanoparticles or impurities in the materials. Thus, for deeply understanding the transport and trapping characteristics of charge carriers within the insulation and their influence on electrical performance, it is very important to measure the space charge characteristics under electric stresses [7-8].

In this paper, Polyimide/TiO $2\left(\mathrm{PI} / \mathrm{TiO}_{2}\right)$ nanocomposite films containing surface modified nano- $\mathrm{TiO}_{2}$ particles by employing silane coupling agent were prepared using in-situ dispersion polymerization process. The space charge distribution in both pure PI film and $\mathrm{PI} / \mathrm{TiO}_{2}$ nanocomposite films were investigated based on the pulsed electro-acoustic method (PEA) at room temperature. Charge dynamics in the materials during the Volts-on and Volts-off were analyzed.

\section{EXPERIMENTAL DETAILS}

\section{A. Materials}

The $\mathrm{TiO}_{2}$ inorganic nanoparticles with an average diameter of $50 \mathrm{~nm}$ were purchased from Haitai Nanomaterials Co. Ltd. Other chemicals, namely, pyromellitic dianhydride (PMDA), and 4, 4' -oxy dianiline (ODA) were furnished from Sinopharm Chemical Reagent Co. Ltd., and Shanghai Sanaisi Reagent Co., respectively, and they were used as received. N, $\mathrm{N}$-dimethylacetamide (DMAc) was purchased from Beijing Chemical Reagent Co., and it was stored over $4 \AA$ molecular sieves prior to use to decrease the adsorbed water content.

\section{B. Fabrication of the polyimide nanocomposites}

To improve dispersion and the interaction between nano$\mathrm{TiO}_{2}$ particles and polymer matrix, the nanoparticles were modified by a coupling agent, namely KH550. The procedures of preparing the $\mathrm{PI} /$ inorganic nanoparticle nanocomposite films were similar to those of $\mathrm{PI} / \mathrm{Al}_{2} \mathrm{O}_{3}$ nanohybrids in the previous literature [9] in which in-situ polymerization was adopted. The modified nano- $\mathrm{TiO}_{2}$ was put into a three-necked roundbottomed flask with some DMAc solvent, and ultrasonic vibrated for $30 \mathrm{~min}$. PMDA was added into the suspension in three parts each after $10 \mathrm{~min}$. Then PAA suspension was stirred for $4 \mathrm{~h}$ at this viscosity. After $4 \mathrm{~h}$, a yellow solution with a low molecular weight poly (amic acid) was obtained. Finally, the mixture was cast onto a cleaned glass plate and was kept still in the vacuum oven at room temperature to let out air completely. The PAA was converted to PI by the thermal imidization and then the $\mathrm{PI} / \mathrm{TiO}_{2}$ nanocomposite films were obtained. For 
comparison, the pure PI film was also prepared by employing the same process.

\section{The PEA testing system}

The schematic diagram for the PEA testing system is shown in Fig.1. The technique has been used widely to observe the space charge in dielectric materials and the detailed principle can be found in literature [10]. All the space charge profiles obtained via deconvolution technique [11] were analyzed as a function of time.

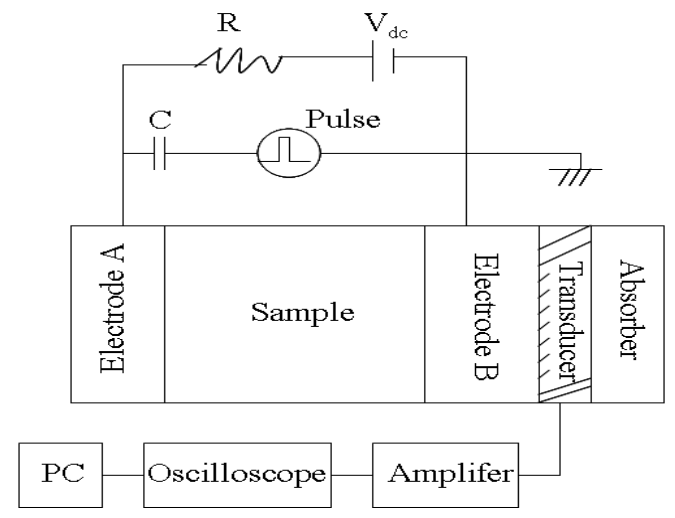

Figure 1. Schematic diagram for the PEA testing system

All the samples were cleaned by alcohol before tested. Each sample was placed between semicon (anode) and aluminum (cathode) electrodes. To improve acoustic coupling and eliminate air gap, a thin layer of silicone oil was formed between the sample and electrodes. In this experiment, the PEA tests were performed on a single layer sample with a thickness of $\sim 60 \mathrm{um}$ at room temperature (see Fig. 1).

\section{RESULTS AND DISCUSSION}

The voltage was applied to the sample for $1 \mathrm{~h}$ and space charge distributions were measured at various times. During this process, the experiment consists of two parts: "volts-on" and "volts-off". "Volts-off" measurements are measurements which are taken immediately after the removal of the external voltage during stressing period. After $1 \mathrm{~h}$ dc stressing, charge decay after the permanent removal of the applied voltage was monitored for further $1 \mathrm{~h}$. However, charge decay results will not be presented and analyzed in this paper due to the limit on paper length. The voltage level was about $2 \mathrm{kV}$, resulting in that the applied electric field was about $33 \mathrm{kV} / \mathrm{mm}$.

\section{A. The Volts-on space charge profiles}

Fig. 2 shows space charge distributions in the samples containing different concentration of the nano- $\mathrm{TiO}_{2}$ particles for $30 \mathrm{~s}, 10 \mathrm{~min}, 30 \mathrm{~min}$ and $60 \mathrm{~min}$, respectively. Electrodes positions are marked by dotted lines in Fig. 2 (a), (b) and (c). At a voltage of $2 \mathrm{kV}$ charge can be found forming in all the samples. In pure PI sample charge from both electrodes have been observed but the amount of the injected charge from the cathode seems more. The charge formation in samples with nano- $\mathrm{TiO}_{2}$ particles is different from the pure PI sample and heterocharges are formed adjacent to the cathode, indicating the influence of the nano- $\mathrm{TiO}_{2}$ particles on charge formation. It also can be seen that the amount of charge in the sample increases with the duration of the applied voltage. Generally, both charge injection and ionization process which is related to the nano- $\mathrm{TiO}_{2}$ particles may occur in the samples. Actually, the injection can be seen in the region adjacent to the anode but the amount of charge is small as it may be masked by charges from ionization. Generally speaking, the formation of homocharge is associated with charge injection from electrodes and hetrocharge with ionization. Observation of hetrocharge formation in nanocomposites may be caused by either ionization of $\mathrm{TiO}_{2}$ or impurities which are difficult to be ruled out completely. Similar results have been observed in $\mathrm{Al}_{2} \mathrm{O}_{3}$ nanoparticles filled epoxy resin system [5].
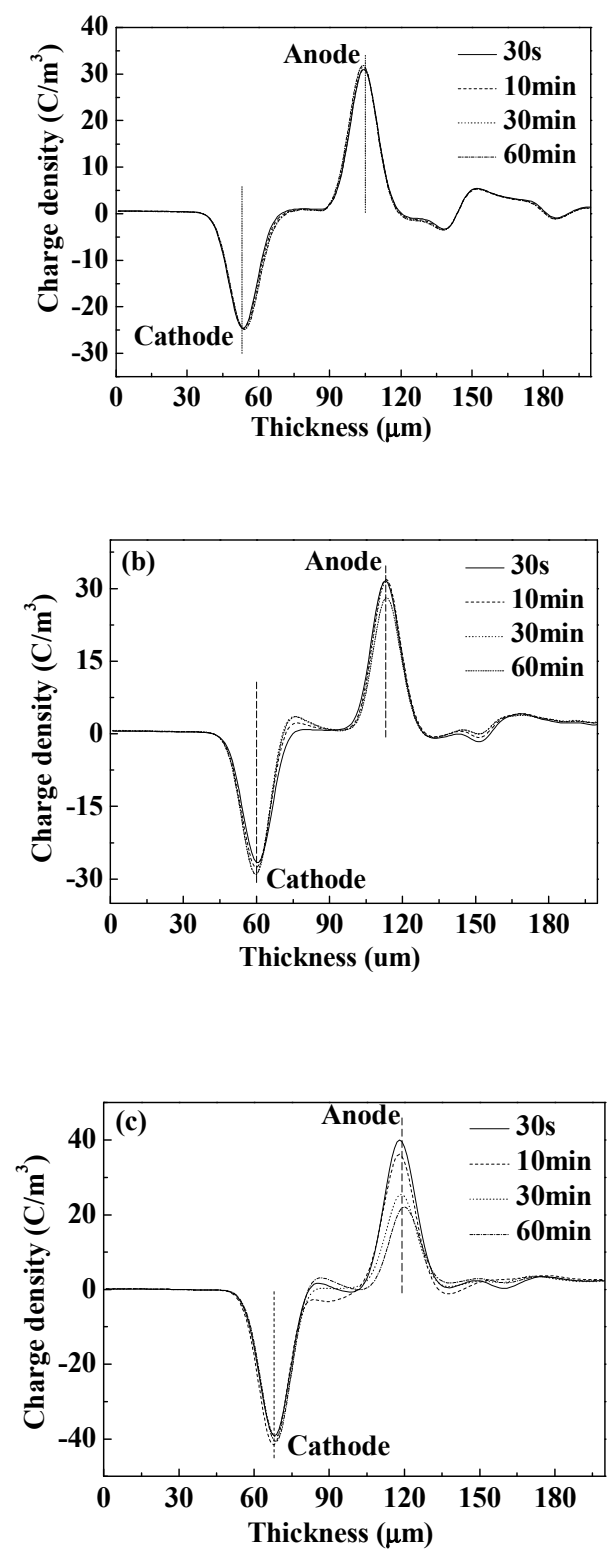

Figure 2. Space charge distribution build-up in different samples at $2 \mathrm{kv}$ : (a) pure PI film, (b) $\mathrm{PI} / \mathrm{TiO}_{2}-15 \mathrm{wt} . \%$ nanocomposite film, (c) $\mathrm{PI} / \mathrm{TiO}_{2}-20 \mathrm{wt} . \%$ nanocomposite film, respectively. 


\section{B. The Volts-off space charge profiles}

On the whole, charges in the sample can be considered consisting of slow charges and fast charges. The volts-on space charge profiles include both slow and fast charges while the volts-off (short-circuit condition) space charge profiles include slow charge [11].
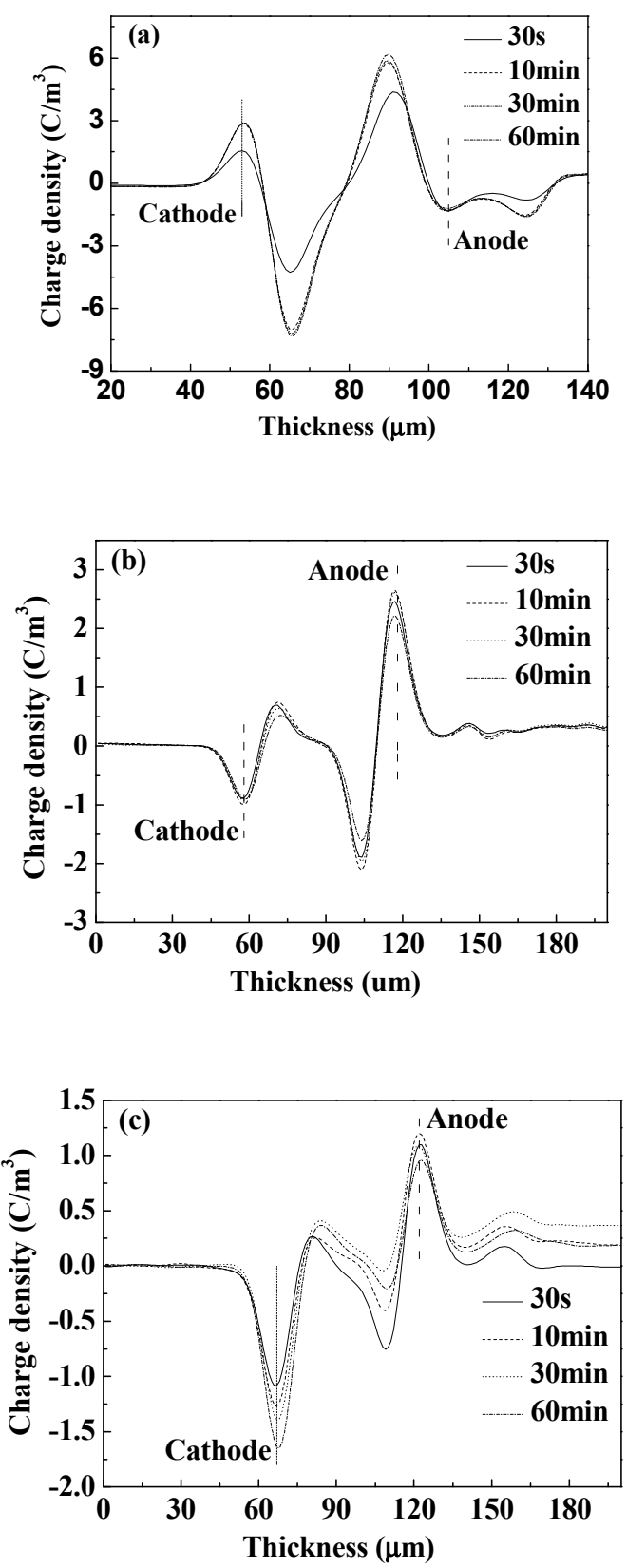

Figure 3. Space charge distribution after removing the stress in the films: (a)pure PI film, (b) $\mathrm{PI} / \mathrm{TiO}_{2}-15$ wt.\% nanocomposite film and (c) $\mathrm{PI} / \mathrm{TiO}_{2}-20$ wt. $\%$ nanocomposite film, respectively

Fig. 3 shows space charge distribution of different samples from "volts-off" measurement taken at various times after volts-on test. The volts-off space charge profiles show trapped charge in the sample after the applied voltage has been removed. In the pure PI sample, significant amount of negative and positive charges remain in the regions close to the electrodes and the amount of charge increases with the duration of voltage application, indicating bipolar injection. This is in agreement with volts-on measurement. For nanocomposites the strongest feature is the observation of heterocharge peak in the vicinity of the anode electrode, which is not seen with volts-on measurements. It is possible that the observed heterocharge near to the anode is masked by the injected positive charge during volts-on. The presence of heterocharges is often associated with ionization taken place in the material. In view of Fig. 3(a) where bipolar charge injection has occurred, there may be two processes competing with the sample. The PEA technique only reveals the net charge. It is worthy of notice that amount of heterocharge adjacent to the cathode is small compared with that near to the anode. There are two possible reasons. Firstly, the introduction of the nano- $\mathrm{TiO}_{2}$ particles may hinder charge injection on both electrodes but the extent may be different. On the other hand, it is also possible that injected positive and negative charges may have different mobilities. This will be further discussed in the next section. At the present stage it is not clear whether the ionization is caused by the nano- $\mathrm{TiO}_{2}$ particles, coupling agent or impurity associated with nano- $\mathrm{TiO}_{2}$ introduction. Another feature is the amount of heterocharge in the nanocomposite with 20 wt. $\%$ nano- $\mathrm{TiO}_{2}$ particles is less than that with 15 wt.\% nano- $\mathrm{TiO}_{2}$ particles. The exact mechanism is not known. We believe there a competing process together with some interaction among the injected charge, inoization and the influence of the nano- $\mathrm{TiO}_{2}$ particles on charge injection.

\section{The accumulated space charge profiles}

PEA only shows the total charge. The accumulated bulk space charge $\rho(x)$ in a sample can be expressed using the following equation [12]:

$$
\rho(x)=\rho_{\text {volts-on }}(x)-a \rho_{\text {ref }}(x)
$$

where $\rho_{\text {volts-on }}(x)$ is the volts-on space charge profile, $\rho_{\text {ref }}(x)$ is the charge induced by the applied voltage as a reference signal for calibration, $a$ is a ratio of the applied voltage over the reference voltage. Assuming that there is no fast charge in the sample, $\rho(x)$ will be equal to $\rho_{\text {volts-off }}(x)$. Namely

$$
\rho_{\text {fast }}(x)=\rho(x)-\rho_{\text {volts }-o f f}(x) \approx 0
$$

where $\rho_{\text {fast }}(x)$ is the fast charge.

In the measurement results presented in this paper, a short time interval was needed between the removal of the applied voltage till the measurement of $\rho_{\text {volts-off }}(x)$. The time interval for such measurement was about $10 \sim 15 \mathrm{sec}$. The fast charges could liberate themselves during this time and leak away from the sample. 

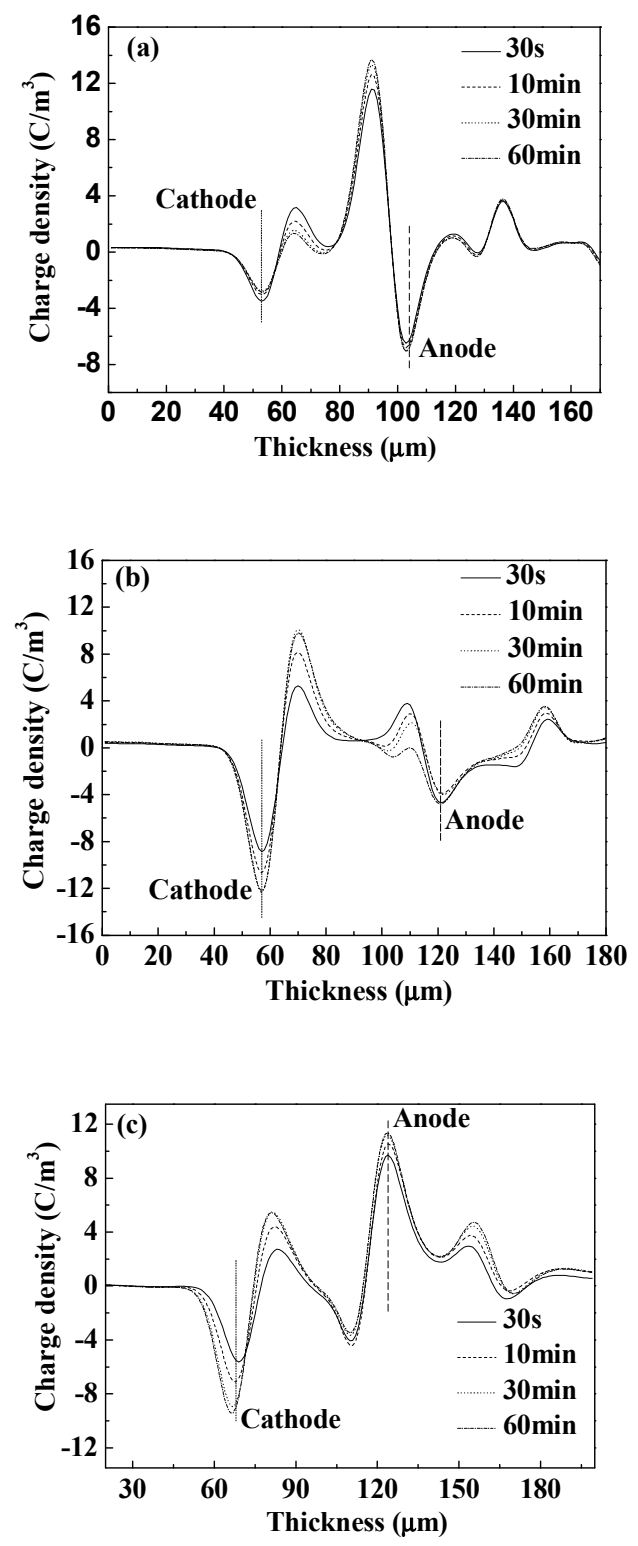

Figure 4. Space charge distribution related to the charges $\left(\rho_{\text {volts }-o n}(x)-a \rho_{\text {ref }}(x)\right)$ in the films: (a)pure PI film, (b) $\mathrm{PI} / \mathrm{TiO}_{2}-15$ wt.\% nanocomposite film and (c) $\mathrm{PI} / \mathrm{TiO}_{2}-20$ wt.\% nanocomposite film, respectively

Fig. 4 shows space charge distributions related to the charge $\left(\rho_{\text {volts-on }}(x)-a \rho_{\text {ref }}(x)\right)$ for different samples. For the pure PI sample, positive charge can be seen dominating, meaning a high mobility of positive charge. This can be further observed by increasing positive charge magnitude adjacent to the cathode in Fig. 4(b). This may be the reason for the observation in Fig. 3 (b). The reduction of positive charge peak close to the anode supports that the addition of nano- $\mathrm{TiO}_{2}$ particle can suppress charge injection. More evidence of suppression on charge injection can be seen from Fig. 4(c).

\section{CONCLUSION}

In summary, polyimide nanocomposite films loaded with different concentration of the nano- $\mathrm{TiO}_{2}$ particles were fabricated using in-situ dispersive polymerization. The space charge dynamics in these nanocomposite films have been investigated by using the PEA technique. The main conclusions are as follows.

Bipolar charge injection has taken place in pure PI sample. The amount of space charge injected increases with the duration of applied voltage. The space charge characteristics in nanocomposites containing the nano- $\mathrm{TiO}_{2}$ particles are very different from the pure PI sample. Heterocharges dominate the distribution indicating ionization occurrence.

The addition of the nano- $\mathrm{TiO}_{2}$ particles into PI matrix may also hinder charge injection process. As space charge dynamics are closely related to the electrical performance, it is anticipated that the electrical performance of polyimide based nanocomposites will be different from the pure PI polymer. This will be carried out in our future research.

\section{REFERENCES}

[1] P. C. Chiang, W. T. Whang. "The synthesis and morphology characteristic study of BAO-ODPA polyimide/ $\mathrm{TiO}_{2}$ nano hybrid films", Polymer, Vol. 44, pp.2249-2254, 2003.

[2] W. L. Qiu, Y. J. Luo, F. T. Chen, Y. Q. Duo, H. M. Tan, "Morphology and size control of inorganic particles in polyimide hybrids by using $\mathrm{SiO}_{2}-\mathrm{TiO}_{2}$ mixed oxide", Polymer, Vol. 44, pp: 5821-5826, 2003.

[3] Y. H. Zhang, S. G. Lu, Y. Q. Li, Z. M. Dang, J. H. Xin, S. Y. Fu, G. T. Li, R. R. Guo, L. F. Li, “ Novel Silica Tube/Polyimide Composite Films with Variable Low Dielectric Constant”, Adv. Mater., Vol. 17, pp. 10561059, 2005.

[4] T. Tanaka, G.C. Montanari and R. Mulhaupt, "Polymer Nanocomposites as Dielectrics and Electrical Insulation-perspectives for Processing Technologies, Material Characterization and Future Applications", IEEE Trans. Dielectr. Electr. Insul., Vol. 11, pp. 763-784, 2004.

[5] A. Hajiyiannis and G. Chen, "Space cahrge formation in epoxy resin including various nanofillers", Annual report conf. Electr. Insul. Dielec. Phenom., pp. 714-717, 2008.

[6] L. Dissado, G. Mazzanti and G. C. Montanari, "The incorporation of space charge degradation in the life model for electrical insulating materials", IEEE Trans. Dielectr. Electr. Insul., Vol.2, pp. 1147-1158, 1995.

[7] G. Chen, Y. Tanaka, T. Takada, and L. zhong, "Effect of polyethylene interface on space charge formation", IEEE Transl. Dielec. Electr. Insul., Vol. 11, pp. 113-121,2004.

[8] G. Chen, M. Fu, X. Z. Liu, and L. S. Zhong, "Ac aging and space-charge characteristic in low-density polyethylene polymeric insulation", J. Appl. Phys., Vol. 97, pp. 083713-1-4,2005.

[9] H. Li, G. Liu, B. Liu, W. Chen, and S. Chen, "Dielectric properties of polyimide/Al2O3 hybrids synthesized by in-situ polymerization", Materials Lett., Vol. 61, pp: 1507-1511,2007.

[10] T. Maeno, T. Futami, H. Kushibe, T. Takada, and C. M. Cooke, "Measurement of spatial charge distribution in thick dielectrics using the pulsed electroacoustic method", IEEE Transl. Electr. Insul., Vol. 23, pp. 433-439,1988.

[11] G. Chen, Y. L. Chong, and M. Fu, "Calibration of the pulsed electroacoustic technique in the presence of trapped charge", Measu. Sci. Techn., Vol. 17, pp. 1974-1980, 2006.

[12] Y. L. Chong, G. Chen, and Y. F. F. Ho, "Temperature effect on space charge dynamics in XLPE insulation", IEEE Trans. Dielectr. Electr. Insul., Vol. 14, pp. 65-76, 2007. 